\title{
Towards a new paradigm for heavy-light meson spectroscopy
}

\author{
Meng-Lin Du, ${ }^{1}$ Miguel Albaladejo, ${ }^{2}$ Pedro Fernandez-Soler, ${ }^{3}$ Feng-Kun Guo, ${ }^{4,5,}{ }^{*}$ Christoph Hanhart, ${ }^{6}$ \\ Ulf-G. Meißner, ${ }^{1,6}$ Juan Nieves, ${ }^{3}$ and De-Liang Yao ${ }^{3}$ \\ ${ }^{1}$ Helmholtz-Institut für Strahlen- und Kernphysik and Bethe Center for Theoretical Physics, \\ Universität Bonn, D-53115 Bonn, Germany \\ ${ }^{2}$ Departamento de Física, Universidad de Murcia, E-30071 Murcia, Spain \\ ${ }^{3}$ Instituto de Física Corpuscular (IFIC), Centro Mixto CSIC-Universidad de Valencia, \\ Institutos de Investigación de Paterna, Apartado 22085, E-46071 Valencia, Spain \\ ${ }^{4}$ CAS Key Laboratory of Theoretical Physics, Institute of Theoretical Physics, \\ Chinese Academy of Sciences, Beijing 100190, China \\ ${ }^{5}$ School of Physical Sciences, University of Chinese Academy of Sciences, Beijing 100049, China \\ ${ }^{6}$ Institute for Advanced Simulation, Institut für Kernphysik and Jülich Center for Hadron Physics, \\ Forschungszentrum Jülich, D-52425 Jülich, Germany
}

(Received 24 July 2018; published 20 November 2018)

Since 2003 many new hadrons, including the lowest-lying positive-parity charm-strange mesons $D_{s 0}^{*}(2317)$ and $D_{s 1}(2460)$, have been observed that do not conform with quark-model expectations. It was recently demonstrated that various puzzles in the charm-meson spectrum find a natural resolution if the SU(3) multiplets for the lightest scalar and axial-vector states, among them the $D_{s 0}^{*}(2317)$ and the $D_{s 1}(2460)$, owe their existence to the nonperturbative dynamics of Goldstone-boson scattering off $D_{(s)}$ and $D_{(s)}^{*}$ mesons. Most importantly the ordering of the lightest strange and nonstrange scalars becomes natural. We demonstrate for the first time that this mechanism is strongly supported by the recent high quality data on the $B^{-} \rightarrow D^{+} \pi^{-} \pi^{-}$provided by the LHCb experiment. This implies that the lowest quark-model positive-parity charm mesons, together with their bottom counterparts, if realized in nature, do not form the ground-state multiplet. This is similar to the pattern that has been established for the scalar mesons made from light up, down, and strange quarks, where the lowest multiplet is considered to be made of states not described by the quark model. In a broader view, the hadron spectrum must be viewed as more than a collection of quark-model states.

DOI: 10.1103/PhysRevD.98.094018

One of the currently most challenging problems in fundamental physics is to understand the nonperturbative regime of Quantum Chromodynamics (QCD), the fundamental theory for the interaction of quarks and gluons. However, since the quark and gluon fields are confined inside color-neutral hadrons, what needs to be achieved is a quantitative understanding of the hadron spectrum. Firstprinciple lattice QCD (LQCD) calculations are indispensable in this regard. In many cases, one can efficiently bridge the computationally intensive LQCD framework with complicated experimental observables using chiral perturbation theory (ChPT) - the effective field theory for QCD at low energies-and its unitarization to fulfill probability conservation. In this work we demonstrate

\footnotetext{
*fkguo@itp.ac.cn
}

Published by the American Physical Society under the terms of the Creative Commons Attribution 4.0 International license. Further distribution of this work must maintain attribution to the author(s) and the published article's title, journal citation, and DOI. Funded by SCOAP ${ }^{3}$. how such a combination leads to the resolution of a number of long-standing puzzles in charm-meson spectroscopy. It also paves the way towards a new paradigm in the spectroscopy for heavy-light mesons.

Until the beginning of the millennium heavy-hadron spectroscopy was assumed to be well understood by means of the quark model [1,2], which describes the positiveparity ground-state charm mesons as bound systems of a heavy quark and a light antiquark in a $P$-wave. This belief was put into question in 2003, when the charm-strange scalar $\left(J^{P}=0^{+}\right)$and axial-vector $\left(1^{+}\right)$mesons $D_{s 0}^{*}(2317)$ [3] and $D_{s 1}(2460)$ [4] were discovered (for recent reviews on new hadrons, see Refs. [5-11]), since the states showed properties at odds with the quark model. Moreover, attempts to adjust the quark model raised more questions [12]. Various alternative proposals were put forward about the nature of these new states including $D^{(*)} K$ hadronic molecules (loosely bound states of two colorless hadrons) $[13,14]$, tetraquarks (compact states made of two quarks and two antiquarks) [15], and chiral partners (doublets due to the chiral symmetry breaking of QCD in heavy-light 
systems) [16,17]. The situation became more obscure in 2004, when two new charm-nonstrange mesons, the $D_{0}^{*}(2400)$ [18] and $D_{1}(2430)$ [19], were observed. In brief, the experimental discoveries brought up three puzzles:

(1) Why are the $D_{s 0}^{*}(2317)$ and $D_{s 1}(2460)$ masses much lower than the quark-model expectations for the lowest $0^{+}$and $1^{+} c \bar{s}$ mesons?

(2) Why is the mass difference between the $D_{s 1}(2460)$ and the $D_{s 0}^{*}(2317)$ equal to that between the groundstate vector meson $D^{*+}$ and pseudoscalar meson $D^{+}$ within $2 \mathrm{MeV}$ ?

(3) Why are the $D_{0}^{*}(2400)$ and $D_{1}(2430)$ masses almost equal to or even higher than their strange siblings, a relationship exploited in many works [20-23], although states with an additional strange quark are typically at least $100 \mathrm{MeV}$ heavier since $m_{s} / m_{d} \simeq 20$, see, e.g., Ref. [24]?

Although their bottom cousins are still being searched for in high-energy experiments, it is natural to ask whether such puzzles will be duplicated there and in other sectors.

As outlined below, in recent works it was demonstrated that analyses combining effective field theory methods with LQCD allows one to resolve all those puzzles. These analyses suggest that all low-lying positive-parity heavy open-flavor mesons qualify as hadronic molecules. In this paper we add two crucial pieces to the existing line of reasoning, namely we propose a lattice QCD study at unphysical quark masses that will allow one to see the twomeson character of the mentioned states more explicitly, and we demonstrate that recent data on $B^{-} \rightarrow D^{+} \pi^{-} \pi^{-}$ show a nontrivial structure fully in line with the proposed dynamical picture.

One reason why the analyses that led to the $D_{0}^{*}(2400)$ and $D_{1}(2430)$ resonance parameters in the Review of Particle Physics (RPP) [24] should be questioned is that the amplitudes used were inconsistent with constraints from the chiral symmetry of QCD. As its chiral symmetry is spontaneously broken, the pions, kaons, and eta arise as Goldstone bosons with derivative and thus energydependent interactions even for $S$-wave couplings. The standard Breit-Wigner (BW) resonance shapes used in the experimental analyses correspond, however, to constant couplings. Moreover, the energy range of these states overlaps with various $S$-wave thresholds that necessarily need to be considered in a sound analysis, as these thresholds can leave a remarkable imprint on observables as will be shown below. A theoretical framework satisfying such requirements is provided by the unitarized chiral perturbation theory (UChPT) for heavy mesons [25-34]. In this approach, ChPT at a given order is used to calculate the interaction potential, which is then resummed in a scattering equation to fulfill exact two-body unitarity and allows for the generation of resonances as pioneered in Ref. [35]. Although there is no unique method for unitarization, different methods do not differ much as long as there are no prominent left-hand cuts [36], as is the case here. It should be mentioned that any algebraic unitarization generates logarithms at higher order with wrong coefficients - this is discussed for the case of $\pi \pi$ scattering in Ref. [37] (for a more recent discussion see Ref. [38]). As long as the unitarization is set up as for the amplitudes employed here, those appear only at orders higher than the order of the potential. However, there is no a priori way to estimate their significance and the reliability of the amplitudes must be tested, e.g., by a comparison with lattice data or experiment. We will employ here the next-to-leading order (NLO) version whose free parameters have been fixed to the Goldstone-boson-charm-meson scattering lengths determined in fully dynamical LQCD in channels without disconnected diagrams [32]. Later it was demonstrated [39] that these coupled-channel amplitudes properly predict the energy levels generated in LQCD (with a pion mass $M_{\pi} \simeq 391 \mathrm{MeV}$ ) for the isospin-1/2 channel even beyond the threshold [40]. This means that now the scattering amplitudes for the coupled Goldstone-bosoncharm-meson system are available that are based on QCD. Moreover, those amplitudes allow us to identify the poles in the complex energy plane reflecting the lowest positiveparity meson resonances of QCD in the charm sector as well as in the bottom sector, once heavy quark flavor symmetry [41] is employed. The predicted masses for the lowest charm-strange positive-parity mesons are fully in line with the well-established measurements, and those for the bottom-strange mesons are consistent with LQCD results with an almost physical pion mass [42], see Table I where the uncertainties quoted stem from the one-sigma uncertainties of the parameters in the NLO UChPT determined in Ref. [32].

It should be noted that in principle in addition to the mentioned uncertainty there is potentially an additional uncertainty stemming from the truncation of the chiral expansion at NLO-in other words terms of order $\left(M_{K} / \Lambda_{\chi}\right)^{3} \sim 10 \%$ were neglected. It is difficult to translate this uncertainty into an uncertainty of the pole locations since the parameters of the NLO potential were fixed to LQCD data such that higher order effects are effectively absorbed into the parameters. However, we did check that a variation of the strength of the potential by $10 \%$ simply moved the poles by $10-20 \mathrm{MeV}$-but neither the number

TABLE I. Predicted masses of the lowest positive-parity heavystrange mesons in comparison with the measured values [24] and latest $\mathrm{LQCD}$ results, in units of $\mathrm{MeV}$.

\begin{tabular}{cccc}
\hline \hline & Prediction & RPP & LQCD \\
\hline$D_{s 0}^{*}$ & $2315_{-28}^{+18}$ & $2317.7 \pm 0.6$ & $2348_{-4}^{+7}[43]$ \\
$D_{s 1}$ & $2456_{-21}^{+15}$ & $2459.5 \pm 0.6$ & $2451 \pm 4[43]$ \\
$B_{s 0}^{*}$ & $5720_{-23}^{+16}$ & $\ldots$ & $5711 \pm 23[42]$ \\
$B_{s 1}^{*}$ & $5772_{-21}^{+15}$ & $\ldots$ & $5750 \pm 25[42]$ \\
\hline \hline
\end{tabular}


of poles nor their sheets did change as a result of this variation.

The first two of the puzzles listed above are solved in this picture: the $D^{(*)} K$ hadronic molecules do not correspond to the quark-model $c \bar{s}$ states; spin symmetry predicts that the binding energies are independent of the heavy meson spin up to an uncertainty of about $10 \%$, as the leading spin symmetry breaking interaction is also of NLO in the chiral expansion. Moreover, there are two poles, corresponding to two resonances, in the $I=1 / 2$ and strangeness $S=0$ channel. The predicted poles, located at the complex energies $M-i \Gamma / 2$, for both scalar and axial-vector charm and bottom mesons are listed in Table II. The masses for the lower nonstrange resonances are smaller than those for the strange ones, leading to the solution to the third puzzle. For comparison the currently quoted masses and widths of the $D_{0}^{*}(2400)^{0}$ and $D_{1}(2430)^{0}$ given in the RPP are also listed.

This pattern of two $I=1 / 2$ states emerges naturally in the underlying formalism since already leading order ChPT interactions are attractive in two flavor multiplets to which the two states belong: the antitriplet and the sextet $[25,39]$. These two scalar isospin $I=1 / 2$ states were predicted in the earlier works of Refs. [25,26], where, however, less refined amplitudes were employed.

Given the above discussion, it is important to test the scenario outlined above as much as possible. In this work we discuss two possible paths: On the one hand we propose a numerical experiment on the lattice, and on the other hand we demonstrate that recent experimental data provide additional support of the nontrivial dynamics that leads to the existence of the light positive-parity open-charm states.

If the mentioned states were $\bar{q} c$ states, they would all be members of the flavor antitriplet-the presence of the sextet is a nontrivial prediction emerging from the meson-meson dynamics that the picture presented above is based on. We notice that while we predicted two $I=1 / 2$ states (see Table II), the Hadron Spectrum Collaboration reported only one, located just below the $D \pi$ threshold, in their lattice calculation at $M_{\pi} \simeq 391 \mathrm{MeV}$ [40]. This is in line with the expectation that the lower pole would become a bound state at $M_{\pi} \gtrsim 350 \mathrm{MeV}$ [32]. The authors of Ref. [40] report that they "do not find any further poles in

TABLE II. Predicted poles corresponding to the positive-parity heavy-light nonstrange mesons given as $(M, \Gamma / 2)$, with $M$ the mass and $\Gamma$ the total decay width, in units of $\mathrm{MeV}$. The current RPP [24] values are listed in the last column.

\begin{tabular}{cccc}
\hline \hline & Lower pole & Higher pole & RPP \\
\hline$D_{0}^{*}$ & $\left(2105_{-8}^{+6}, 102_{-11}^{+10}\right)$ & $\left(2451_{-26}^{+35}, 134_{-8}^{+7}\right)$ & $(2318 \pm 29,134 \pm 20)$ \\
$D_{1}$ & $\left(2247_{-6}^{+5}, 107_{-10}^{+11}\right)$ & $\left(2555_{-30}^{+47}, 203_{-9}^{+8}\right)$ & $\left(2427 \pm 40,192_{-55}^{+65}\right)$ \\
$B_{0}^{*}$ & $\left(5535_{-11}^{+9}, 113_{-17}^{+15}\right)$ & $\left(5852_{-19}^{+16}, 36 \pm 5\right)$ & $\ldots$ \\
$B_{1}$ & $\left(5584_{-11}^{+9}, 119_{-17}^{+14}\right)$ & $\left(5912_{-18}^{+15}, 42_{-4}^{+5}\right)$ & $\ldots$ \\
\hline \hline
\end{tabular}

the region where ... [their lattice analysis] constrained the amplitudes." This does on the other hand not exclude the presence of the second pole advocated for in Ref. [39] as well as above: The quote simply reflects the fact that while various of the amplitudes employed in the analysis of Ref. [40] contained a second pole, its location was strongly parametrization dependent [44]. With the quark masses used in Ref. [40], the predicted sextet pole is located deep in the complex plane [39], and thus it is not captured easily. The advantage of our amplitudes compared to those employed in the analysis of Ref. [40] is that they are constrained by both the pattern of chiral symmetry breaking of QCD as well as lattice data in additional channels. To further test our explanation for the light positive-parity open-charm states, we propose to search for them in lattice studies at an $\mathrm{SU}(3)$ symmetric point, with a relatively large quark mass $m_{u}=m_{d}=m_{s}$, such that the lightest pseudoscalar-meson masses will be near or above $m_{\phi} \gtrsim 475 \mathrm{MeV}$. We predict that the sextet pole will become a virtual state below threshold for such large quark masses, and that it would even become a bound state for higher quark masses. This behavior is illustrated in Fig. 1, where one can see that now the pole is close to threshold, and it should be easy to detect in a lattice calculation. Note that the trajectory of the pole displayed in Fig. 1, in particular that in a certain parameter range resonance poles exist in the complex energy plane below threshold, is common for two-meson states in a relative $S$-wave. This feature is discussed in quite general terms in Ref. [45] [see also Refs. [46,47] for the $f_{0}(500)$ case] and was first presented for the open-flavor states in the focus here in Ref. [31].

In the following, we show that our resolution to these puzzles is backed by precise experimental data by showing that the amplitudes with the two $D_{0}^{*}$ states are fully consistent with the LHCb measurements of the reaction

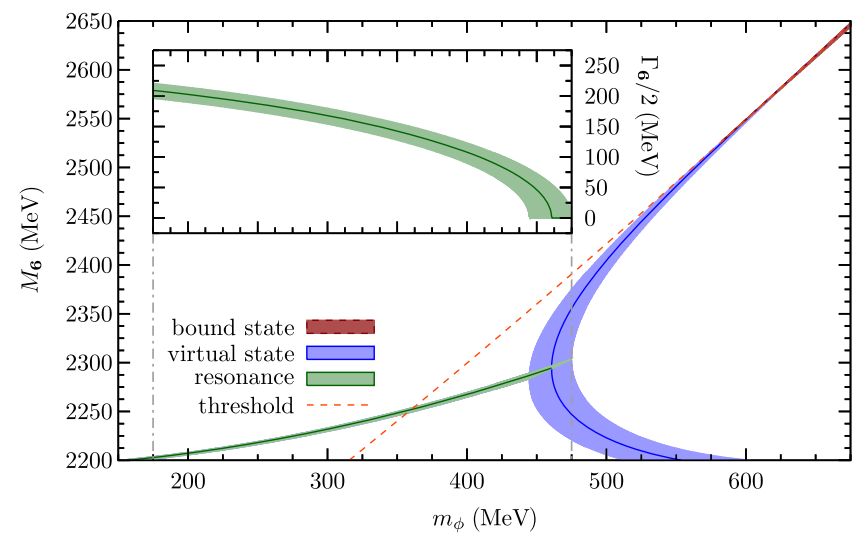

FIG. 1. Illustration of the mass of the predicted sextet state at the $\mathrm{SU}(3)$ symmetric point as a function of the Goldstone-boson mass $m_{\phi}$. Below $m_{\phi} \lesssim 475 \mathrm{MeV}$, the pole is a resonance with its imaginary part $\left(\Gamma_{6} / 2\right)$ shown in the inserted subfigure. Above $m_{\phi} \simeq 475 \mathrm{MeV}$, it evolves into a pair of virtual states, and finally it becomes a bound state at $m_{\phi} \simeq 600 \mathrm{MeV}$. 


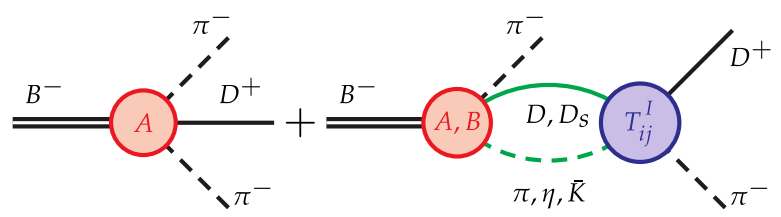

FIG. 2. The decay amplitude for $B^{-} \rightarrow D^{+} \pi^{-} \pi^{-}$. Here, $A, B$ parametrize the production vertex, see Eq. (2), and $T_{i j}^{I}$ denotes the final state interactions between the charm and light mesons.

$B^{-} \rightarrow D^{+} \pi^{-} \pi^{-}$[48], which are at present the best data providing access to the $D \pi$ system and thus to the nonstrange scalar charm mesons. Therefore, all the available theoretical, experimental, and LQCD knowledge is consistent with the existence of two $D_{0}^{*}$ states in the mass region where there was believed to be only one $D_{0}^{*}(2400)$.

The Feynman diagrams for the decay amplitude for $B^{-} \rightarrow D^{+} \pi^{-} \pi^{-}$are shown in Fig. 2. All the channels $\left(D^{+} \pi^{-}, D^{0} \pi^{0}, D^{0} \eta\right.$, and $\left.D_{s}^{+} K^{-}\right)$coupled to $D^{+} \pi^{-}$need to be considered in the intermediate states. The decay amplitude in the energy region up to $2.6 \mathrm{GeV}$, which is sufficient to study the low-lying scalar states, can be decomposed into $S-, P$-, and $D$-waves,

$$
\mathcal{A}\left(B^{-} \rightarrow D^{+} \pi^{-} \pi^{-}\right)=\sum_{L=0}^{2} \sqrt{2 L+1} \mathcal{A}_{L}(s) P_{L}(z),
$$

where $s$ is the $D^{+} \pi^{-}$center-of-mass energy squared, $\mathcal{A}_{0,1,2}(s)$ correspond to the amplitudes with $D^{+} \pi^{-}$in the $S_{-}, P_{-}$, and $D$-waves, respectively, and $P_{L}(z)$ are the Legendre polynomials. For the $P$ - and $D$-wave amplitudes we use the same BW form as in the LHCb analysis [48]. However, for the $S$-wave we employ

$$
\begin{aligned}
\mathcal{A}_{0}(s)= & A\left\{E_{\pi}\left[2+G_{1}(s)\left(\frac{5}{3} T_{11}^{1 / 2}(s)+\frac{1}{3} T^{3 / 2}(s)\right)\right]\right. \\
& \left.+\frac{1}{3} E_{\eta} G_{2}(s) T_{21}^{1 / 2}(s)+\sqrt{\frac{2}{3}} E_{\bar{K}} G_{3}(s) T_{31}^{1 / 2}(s)\right\} \\
& +B E_{\eta} G_{2}(s) T_{21}^{1 / 2},
\end{aligned}
$$

where $A$ and $B$ are two independent couplings following from SU(3) flavor symmetry [49], and $E_{\pi, \eta, \bar{K}}$ are the energies of the light mesons. The effective Lagrangian for the production vertex leading to the above amplitude can be found in the Appendix. Here the $T_{i j}^{I}(s)$ are the $S$-wave scattering amplitudes for the coupled-channel system with total isospin $I$, where $i, j$ are channel indices with 1,2 , and 3 referring to $D \pi, D \eta$, and $D_{s} \bar{K}$, respectively. These scattering amplitudes can be found in Ref. [32] where also all the parameters were fixed. The unitarity relation

$$
\operatorname{Im} \mathcal{A}_{0, i}(s)=-\sum_{j} T_{i j}^{*}(s) \rho_{j}(s) \mathcal{A}_{0, j}(s)
$$

with $\rho_{j}(s)$ the two-body phase space factor in channel $j$, is satisfied as long as $\operatorname{Im} G_{i}(s)=-\rho_{i}(s)$, which allows us to represent $G_{i}(s)$ via a once-subtracted dispersion relation [50]. The same subtraction constant $a_{A}$ is taken for all channels. The amplitude of Eq. (2) embodies chiral symmetry constraints and coupled-channel unitarity, and thus has a sound theoretical foundation. Here the final state interaction between the two $\pi^{-}$mesons is neglected because the two pions are in an isospin-tensor state, and they have a large relative momentum so that they quickly fly away from each other.

The so-called angular moments, see, e.g., Refs. [48,51], contain important information about the partial-wave phase variations. Neglecting partial waves with $L \geq 3$, which is perfectly fine in the energy region of interest as indicated by the $\mathrm{LHCb}$ data, the first few moments are given by

$$
\begin{aligned}
\left\langle P_{0}\right\rangle & \propto\left|\mathcal{A}_{0}\right|^{2}+\left|\mathcal{A}_{1}\right|^{2}+\left|\mathcal{A}_{2}\right|^{2}, \\
\left\langle P_{2}\right\rangle & \propto \frac{2}{5}\left|\mathcal{A}_{1}\right|^{2}+\frac{2}{7}\left|\mathcal{A}_{2}\right|^{2}+\frac{2}{\sqrt{5}}\left|\mathcal{A}_{0}\right|\left|\mathcal{A}_{2}\right| \cos \left(\Delta \delta_{2}\right), \\
\left\langle P_{13}\right\rangle & \equiv\left\langle P_{1}\right\rangle-\frac{14}{9}\left\langle P_{3}\right\rangle \propto \frac{2}{\sqrt{3}}\left|\mathcal{A}_{0}\right|\left|\mathcal{A}_{1}\right| \cos \left(\Delta \delta_{1}\right),
\end{aligned}
$$

where $\Delta \delta_{1,2}$ are the phase differences of $P$ - and $D$-waves relative to the $S$-wave, respectively. Instead of $\left\langle P_{1}\right\rangle$ and $\left\langle P_{3}\right\rangle$ we propose to analyze the linear combination $\left\langle P_{13}\right\rangle$, since it only depends on the $S-P$ interference up to $L=2$ and is particularly sensitive to the $S$-wave phase motion.

We fit to the data of the moments defined in Eq. (4) up to $M_{D^{+} \pi^{-}}=2.54 \mathrm{GeV}$ for the decay $B^{-} \rightarrow D^{+} \pi^{-} \pi^{-}$measured by the LHCb Collaboration [48]. Except for the $S$-wave $D \pi$ given in Eq. (2), we include the resonances $D^{*}$ and $D^{*}(2680)$ in the $P$-wave and $D_{2}(2460)$ in the $D$-wave. Their masses and widths are fixed to the central values in the LHCb analysis [48], and their phase parameters are denoted by $\delta_{D^{*}}, \delta_{D^{*}}^{\prime}$, and $\delta_{D_{2}}$, respectively. The best fit has $\chi^{2} /$ d.o.f. $=1.7$ and the parameter values are $B / A=$ $-3.6 \pm 0.1, a_{A}=1.0 \pm 0.1, \delta_{D^{*}}=-0.42 \pm 0.04, \delta_{D^{*}}^{\prime}=$ $1.1 \pm 0.2$, and $\delta_{D_{2}}=-0.83 \pm 0.07$. We do not show the four normalization parameters (three for these resonances and one for the $S$-wave). A comparison of the best fit with the LHCb data is shown in Fig. 3 together with the best fit provided by the LHCb Collaboration [48] (dashed lines), where cubic splines were used to interpolate between certain anchor points-below we detail this method further. The bands in Fig. 3 reflect the one-sigma errors of the parameters in the scattering amplitudes determined in Ref. [32]. It is worthwhile to notice that in $\left\langle P_{13}\right\rangle$, where the $D_{2}(2460)$ does not play any role, the data show a significant variation between 2.4 and $2.5 \mathrm{GeV}$. Theoretically this feature can now be understood as a signal for the opening of the $D^{0} \eta$ and $D_{s}^{+} K^{-}$thresholds at 2.413 and $2.462 \mathrm{GeV}$, respectively, which leads to two cusps in the amplitude. This effect is amplified by the higher pole 

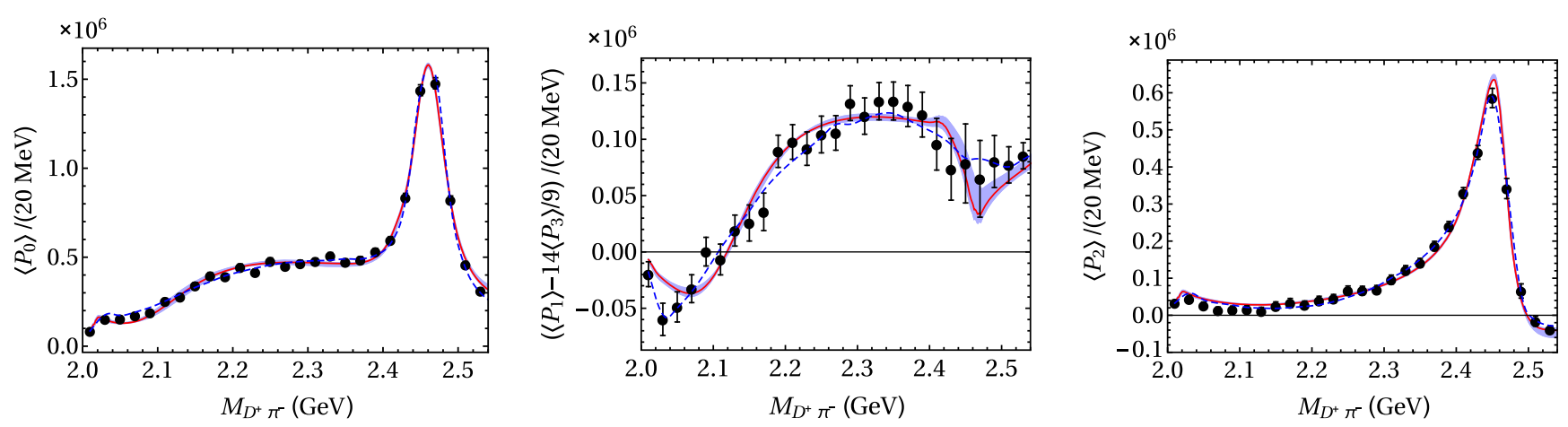

FIG. 3. Fit to the LHCb data for the angular moments $\left\langle P_{0}\right\rangle,\left\langle P_{1}\right\rangle-14\left\langle P_{3}\right\rangle / 9$, and $\left\langle P_{2}\right\rangle$ for the $B^{-} \rightarrow D^{+} \pi^{-} \pi^{-}$reaction [48]. The largest error among $\left\langle P_{1}\right\rangle$ and $14\left\langle P_{3}\right\rangle / 9$ in each bin is taken as the error of $\left\langle P_{1}\right\rangle-14\left\langle P_{3}\right\rangle / 9$. The solid lines show our results, with error bands corresponding to the one-sigma uncertainties propagated from the input scattering amplitudes, while the dashed lines stand for the LHCb fit using cubic splines for the $S$-wave [48].

which is relatively close to the $D_{s} \bar{K}$ threshold on the unphysical sheet.

One might wonder whether the discrepancy between our amplitude and the data for $\left\langle P_{13}\right\rangle$ at low energies points to a deficit of the former. Fortunately the LHCb Collaboration provides more detailed information on their $S$-wave amplitude in Ref. [48]: In the analysis of the data a series of anchor points were defined where the strength and the phase of the $S$-wave amplitude were extracted from the data. Then cubic splines were used to interpolate between these anchor points. In Fig. 4 the $S$-wave amplitude fixed as described above is compared to the LHCb anchor points. Not only does this figure show very clearly that the strength of the $S$-wave amplitude largely determined by the fits to

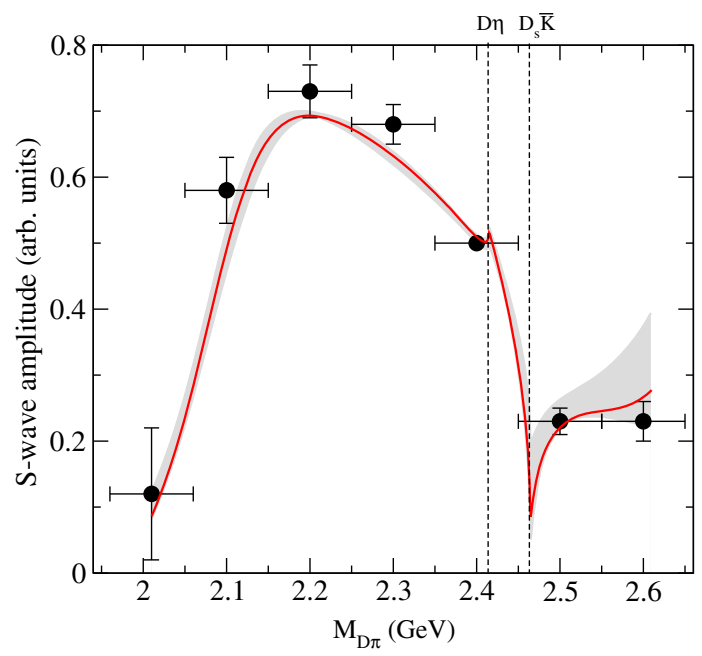

FIG. 4. Comparison of the $S$-wave amplitude determined in this work to the $S$-wave anchor points found in the experimental analysis, shown as the data points [48]. The red line gives the best fit results and the gray band quantifies the uncertainties that emerged from the fitting procedure. The fitting range extends up to $2.55 \mathrm{GeV}$. The dashed perpendicular lines indicate the locations of the $D \eta$ and $D_{s} \bar{K}$ thresholds, respectively. lattice data is fully consistent with the one extracted from the data for $B^{-} \rightarrow D^{+} \pi^{-} \pi^{-}$, the amplitude fixed in the experiment also shows clear structures at both the $D \eta$ and $D_{s} \bar{K}$ thresholds. From our point of view the most natural explanation of those structures is that they are the mentioned cusps enhanced in impact by the pole located nearby. Thus the comparison of the $S$-wave amplitude extracted by the LHCb Collaboration with our result shows the role of the higher pole in the $I=1 / 2$ and $S=0$ channel even more clearly than the angular moments discussed above. This clearly highlights the importance of a coupled-channel treatment for this reaction. An updated analysis of the LHC Run-2 data is called for to confirm the prominence of the two cusps. Notice that the shape of the $S$-wave is determined by only two real parameters $\left(B / A, a_{A}\right)$, while its phase motion is largely determined from unitarity, Eq. (3).

Furthermore, the data for the angular moments for $B_{s}^{0} \rightarrow$ $\bar{D}^{0} K^{-} \pi^{+}$[51] can be easily reproduced in the same framework with the $\bar{D} \bar{K}$ interaction fixed from Ref. [32] again, which has the $\bar{D}_{s 0}^{*}(2317)$ as a dynamically generated state. We focus on the angular moments as functions of the $\bar{D}^{0} K^{-}$invariant mass which were measured in the $\mathrm{LHCb}$ experiment [51]. The decay mechanism is similar to the one in Fig. 2, and the final state $\bar{D}^{0} K^{-}$can be generated from $\bar{D}^{0} K^{-}, D^{-} \bar{K}^{0}, \bar{D}_{s} \eta$, and $\bar{D}_{s} \pi^{0}$ intermediate states. Considering isospin symmetry, the $S$-wave part of the decay amplitude for this process can be written as

$$
\begin{aligned}
\mathcal{A}_{0}(s)= & E_{K}\left[C+\frac{1}{2}(C+A) G_{1}(s) T_{11}^{0}(s)\right. \\
& \left.+\frac{1}{2}(C-A) G_{1}(s) T_{11}^{1}(s)\right] \\
& -\frac{1}{\sqrt{3}}\left(\frac{3}{2} B-C\right) E_{\eta} G_{2}(s) T_{21}^{0}(s),
\end{aligned}
$$

where $C=\sqrt{2}\left(c_{2}+c_{4}\right) / F$ in terms of the low-energy constants (LECs) in Eq. (A1), the channel labels 1 and 2 
refer to the $\bar{D} \bar{K}$ and $\bar{D}_{s} \eta$ channels, respectively, and the superscript of the $T$-matrix refers to the isospin. Note that the Lagrangian in Eq. (A1) does not yield a term contributing to $B_{s}^{0} \rightarrow \bar{D}_{s} \pi^{0} \pi^{+}$. Taking the central values of $B / A$ and the subtraction constant $a_{A}$ as determined from fitting to the $B^{-} \rightarrow D^{+} \pi^{-} \pi^{-}$data, there is only one free parameter in the $S$-wave amplitude, which is $C / A$ (we choose $A$ to serve as the normalization constant for the $S$-wave contribution). For the $P$ - and $D$-waves, we again take the same BW resonances as the $\mathrm{LHCb}$ analysis [51], i.e., $D_{s}^{*}$ and $D_{s}^{*}(2700)$ for the $P$-wave and $D_{s 2}(2573)$ for the $D$-wave, with their masses and widths fixed to the central values in Ref. [51]. The best fits to the angular moments $\left\langle P_{0}\right\rangle,\left\langle P_{2}\right\rangle$, and $\left\langle P_{1}\right\rangle-14\left\langle P_{3}\right\rangle / 9$ for the LHCb $B_{s}^{0} \rightarrow \bar{D}^{0} K^{-} \pi^{+}$data [51] up to $2.7 \mathrm{GeV}$ leads to $\chi^{2} /$ d.o.f. $=1.6$, and the only free parameter in the $S$-wave amplitude is determined to be $C / A=4.8_{-1.7}^{+3.4}$. A comparison of the fit to the data is shown in Fig. 5.

In summary, we have demonstrated that amplitudes fixed from QCD inputs for the Goldstone-boson scattering off charm mesons not only resolve some long-standing puzzles in charm-meson spectroscopy but also are at the same time fully consistent with recent $\mathrm{LHCb}$ data on $B$ decays, which provide by far the most precise experimental information for the $D \pi$ system. The amplitudes have a pole corresponding to the $D_{s 0}^{*}(2317)$ in the isoscalar strangeness $S=1$ channel, and two poles in the $I=1 / 2$ nonstrange channel [39]. The latter pair of poles should replace the lowest $J^{P}=0^{+}$charm nonstrange meson, $D_{0}^{*}(2400)$, listed in the RPP [24]. Similarly, the broad $D_{1}(2430)$ listed in the RPP should also be replaced by two $J^{P}=1^{+}$states.

It should be stressed that the observation that certain scattering amplitudes employ poles does not necessarily imply that the corresponding states need to be interpreted as molecular states. However, the $S$-wave molecular admixture of a near-threshold state can be quantified from the scattering length directly [52]. Applying this argument to the $D K$ scattering length in the $D_{s 0}^{*}(2317)$ channel, predicted in Ref. [32] and determined using LQCD [53], reveals that the molecular component of the $D_{s 0}^{*}(2317)$ is larger than $70 \%$, a conclusion confirmed later in Refs. [43,54,55] for both the $D_{s 0}^{*}(2317)$ and the $D_{s 1}(2460)$. All the poles listed in Tables I and II are spin-flavor partners, due to approximate QCD symmetries. Therefore, they should be envisioned as to have the same origin, i.e., hadronic molecules generated from coupledchannel two-hadron chiral dynamics.

Treating other narrow heavy mesons, such as the $D_{1}(2420)$ and the $D_{2}(2460)$, as matter fields leads to additional molecular states such as the $J^{P}=1^{-} D_{s 1}^{*}(2860)$ [56] and its partners. In fact, the interactions of Goldstone bosons with matter fields are relatively weak at low energies because of the chiral symmetry of QCD, even though hadronic molecular states can be still generated. One would expect that the $S$-wave attractive interaction of other hadrons with heavy mesons, not suppressed by chiral symmetry, may produce hadron-hadron states as well, analogous to nuclei. These states are not the exclusive origin of higher resonances, but they are important contributors to the hadron zoo. Given more and more $S$-wave thresholds at higher energies, quark models are expected to become less and less reliable.

We therefore conclude that the long accepted paradigm underlying open-flavor heavy meson spectroscopy that identifies all ground states with $c \bar{q}$ or $b \bar{q}$ quark-model states is no longer tenable. In a broader view, the hadron spectrum must be viewed not as simply a collection of quark-model states, but rather as a manifestation of a more complex dynamics that leads to an intricate pattern of various types of states that can only be understood by a joint effort from experiment, LQCD, and phenomenology. We close the paper by summarizing a few suggestions that will provide further, nontrivial tests of the scenario proposed here:

(a) Measuring the angular moments, in particular $\left\langle P_{1}\right\rangle-14\left\langle P_{3}\right\rangle / 9$, with unprecedented accuracy for the $B \rightarrow D^{(*)} \pi \pi$ and $B \rightarrow D_{s}^{(*)} \bar{K} \pi$ reactions. This can be done at LHCb and Belle-II. We expect to
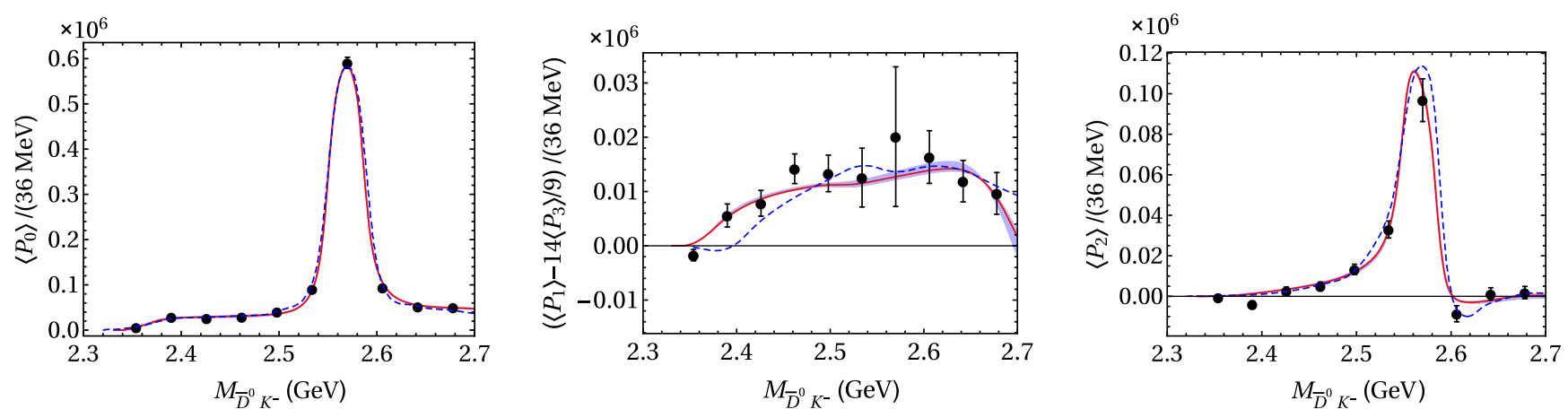

FIG. 5. Fit to the angular moments as a function of the $\bar{D}^{0} K^{-}$invariant mass for the process $B_{s}^{0} \rightarrow \bar{D}^{0} K^{-} \pi^{+}$provided by LHCb [51]. The solid lines represent the present work with the bands corresponding to the one-sigma uncertainty propagated from the input scattering amplitudes, and the dashed lines show the LHCb fit [51]. 
see nontrivial cusp structures at the $D^{(*)} \eta$ and $D_{s}^{(*)} \bar{K}$ thresholds in the former, and near-threshold enhancement in the $D_{s}^{(*)} \bar{K}$ spectrum in the latter [39].

(b) Measuring the hadronic width of the $D_{s 0}^{*}(2317)$, predicted to be of about $100 \mathrm{keV}$ in the molecular scenario [32,57], and much smaller otherwise. This will be measured by the PANDA experiment.

(c) Checking the existence of the sextet pole in LQCD with a relatively large $\mathrm{SU}(3)$ symmetric quark mass.

(d) Searching for the predicted analogous bottom positiveparity mesons both experimentally and in LQCD.

\section{ACKNOWLEDGMENTS}

We acknowledge Tim Gershon, Jonas Rademacker, Mark Peter Whitehead, and Yan-Xi Zhang for discussions on the LHCb data, and thank Sinéad Ryan and David Wilson for communications regarding the LQCD calculation carried out in Ref. [40]. This work is partially supported by the National Natural Science Foundation of China (NSFC) and Deutsche Forschungsgemeinschaft (DFG) through funds provided to the Sino-German Collaborative Research Center "Symmetries and the Emergence of Structure in QCD" (NSFC Grant No. 11621131001, DFG Grant No. TRR110), by the NSFC (Grant No. 11747601), by the Thousand Talents Plan for Young Professionals, by the CAS Key Research Program of Frontier Sciences (Grant No. QYZDB-SSWSYS013), by the CAS Key Research Program (Grant No. XDPB09), by the CAS President's International Fellowship Initiative (PIFI) (Grant No. 2018DM0034), by the CAS Center for Excellence in Particle Physics (CCEPP), by Spanish Ministerio de Economa y Competitividad and the European Regional Development Fund under Contracts No. FIS2014-51948-C2-1-P, No. FPA2016-77313-P, No. FIS2017-84038-C2-1-P, and No. SEV- 2014-0398, by Generalitat Valenciana under Contract No. PROMETEOII/2014/0068, and by the Ayudas para contratos predoctorales para la formacin de doctores program (BES-2015-072049) from the Spanish MINECO and ESF.

\section{APPENDIX: EFFECTIVE LAGRANGIAN}

Here we discuss briefly the effective Lagrangian for the weak decays $\bar{B}$ to $D$ with the emission of two light pseudoscalar mesons, induced by the Cabibbo-allowed transition $b \rightarrow c \bar{u} d$. In the phase space region near the $D \pi$ threshold, chiral symmetry puts constraints on one of the two pions, while the other one moves fast and can be treated as a matter field. Moreover, its interaction with the other particles in the final state can be safely neglected. Then the relevant chiral effective Lagrangian leading to Eq. (2) reads

$$
\begin{aligned}
\mathcal{L}_{\text {eff }}= & \bar{B}\left[c_{1}\left(u_{\mu} t M+M t u_{\mu}\right)+c_{2}\left(u_{\mu} M+M u_{\mu}\right) t\right. \\
& +c_{3} t\left(u_{\mu} M+M u_{\mu}\right)+c_{4}\left(u_{\mu}\langle M t\rangle+M\left\langle u_{\mu} t\right\rangle\right) \\
& \left.+c_{5} t\left\langle M u_{\mu}\right\rangle+c_{6}\left\langle\left(M u_{\mu}+u_{\mu} M\right) t\right\rangle\right] \partial^{\mu} D^{\dagger} .
\end{aligned}
$$

Here $\bar{B}=\left(B^{-}, \bar{B}^{0}, \bar{B}_{s}^{0}\right)$ and $D=\left(D^{0}, D^{+}, D_{s}^{+}\right)$are the fields for bottom and charm mesons, $\langle\cdots\rangle$ denotes the trace in the $\mathrm{SU}(3)$ light-flavor space, and $u_{\mu}=i\left(u^{\dagger} \partial_{\mu} u-u \partial_{\mu} u^{\dagger}\right)$ is the axial current derived from chiral symmetry. The Goldstone bosons are represented nonlinearily via $u=$ $\exp (i \phi /(\sqrt{2} F))$, with

$$
\phi=\left(\begin{array}{ccc}
\frac{1}{\sqrt{2}} \pi^{0}+\frac{1}{\sqrt{6}} \eta & \pi^{+} & K^{+} \\
\pi^{-} & -\frac{1}{\sqrt{2}} \pi^{0}+\frac{1}{\sqrt{6}} \eta & K^{0} \\
K^{-} & \bar{K}^{0} & -\frac{2}{\sqrt{6}} \eta
\end{array}\right),
$$

in terms of the pions $\left(\pi^{ \pm}, \pi^{0}\right)$, the kaons $\left(K^{ \pm}, K^{0}, \bar{K}^{0}\right)$, and the $\eta$, and $F$ denotes the Goldstone-boson decay constant in the chiral limit. In addition, $t=u H u^{\dagger}$ is a spurion field with [49]

$$
H=\left(\begin{array}{lll}
0 & 0 & 0 \\
1 & 0 & 0 \\
0 & 0 & 0
\end{array}\right),
$$

for Cabibbo-allowed decays. The matter field $M$, having the same form as $\phi$, describes the fast moving light meson. The $c_{i}$ $(i=1, \ldots, 6)$ are LECs. This effective Lagrangian considers both chiral, for the regime with soft Goldstone bosons, and SU(3) constraints, the latter of which has been considered in Ref. [49]. In terms of the LECs in the above Lagrangian, the parameters $A$ and $B$ in Eq. (2) can be expressed as $A=$ $\sqrt{2}\left(c_{1}+c_{4}\right) / F$ and $B=2 \sqrt{2}\left(c_{2}+c_{6}\right) /(3 F)$.
[1] M. Gell-Mann, Phys. Lett. 8, 214 (1964).

[2] S. Godfrey and N. Isgur, Phys. Rev. D 32, 189 (1985).

[3] B. Aubert et al. (BABAR Collaboration), Phys. Rev. Lett. 90, 242001 (2003).
[4] D. Besson et al. (CLEO Collaboration), Phys. Rev. D 68, 032002 (2003); 75, 119908(E) (2007).

[5] H. X. Chen, W. Chen, X. Liu, and S. L. Zhu, Phys. Rep. 639, 1 (2016). 
[6] H. X. Chen, W. Chen, X. Liu, Y. R. Liu, and S. L. Zhu, Rep. Prog. Phys. 80, 076201 (2017).

[7] R. F. Lebed, R. E. Mitchell, and E. S. Swanson, Prog. Part. Nucl. Phys. 93, 143 (2017).

[8] A. Esposito, A. Pilloni, and A. D. Polosa, Phys. Rep. 668, 1 (2017).

[9] F.-K. Guo, C. Hanhart, U.-G. Meißner, Q. Wang, Q. Zhao, and B. S. Zou, Rev. Mod. Phys. 90, 015004 (2018).

[10] A. Ali, J. S. Lange, and S. Stone, Prog. Part. Nucl. Phys. 97, 123 (2017).

[11] S. L. Olsen, T. Skwarnicki, and D. Zieminska, Rev. Mod. Phys. 90, 015003 (2018).

[12] R. N. Cahn and J.D. Jackson, Phys. Rev. D 68, 037502 (2003).

[13] T. Barnes, F. E. Close, and H. J. Lipkin, Phys. Rev. D 68, 054006 (2003).

[14] E. van Beveren and G. Rupp, Phys. Rev. Lett. 91, 012003 (2003).

[15] Y.-Q. Chen and X.-Q. Li, Phys. Rev. Lett. 93, 232001 (2004).

[16] W. A. Bardeen, E. J. Eichten, and C. T. Hill, Phys. Rev. D 68, 054024 (2003).

[17] M. A. Nowak, M. Rho, and I. Zahed, Acta Phys. Pol. B 35, 2377 (2004).

[18] J. M. Link et al. (FOCUS Collaboration), Phys. Lett. B 586, 11 (2004).

[19] K. Abe et al. (Belle Collaboration), Phys. Rev. D 69, 112002 (2004).

[20] T. Mehen and R. P. Springer, Phys. Rev. D 72, 034006 (2005).

[21] P. Colangelo, F. De Fazio, F. Giannuzzi, and S. Nicotri, Phys. Rev. D 86, 054024 (2012).

[22] M. H. Alhakami, Phys. Rev. D 93, 094007 (2016).

[23] H.-Y. Cheng and F.-S. Yu, Eur. Phys. J. C 77, 668 (2017).

[24] M. Tanabashi et al. (Particle Data Group), Phys. Rev. D 98, 030001 (2018).

[25] E. E. Kolomeitsev and M. F. M. Lutz, Phys. Lett. B 582, 39 (2004).

[26] F.-K. Guo, P.-N. Shen, H.-C. Chiang, R.-G. Ping, and B.-S. Zou, Phys. Lett. B 641, 278 (2006).

[27] J. Hofmann and M. F. M. Lutz, Nucl. Phys. A733, 142 (2004).

[28] F.-K. Guo, P.-N. Shen, and H.-C. Chiang, Phys. Lett. B 647, 133 (2007).

[29] D. Gamermann, E. Oset, D. Strottman, and M. J. Vicente Vacas, Phys. Rev. D 76, 074016 (2007).
[30] J. M. Flynn and J. Nieves, Phys. Rev. D 75, 074024 (2007).

[31] F.-K. Guo, C. Hanhart, and U.-G. Meißner, Eur. Phys. J. A 40, 171 (2009).

[32] L. Liu, K. Orginos, F.-K. Guo, C. Hanhart, and U.-G. Meißner, Phys. Rev. D 87, 014508 (2013).

[33] M. Altenbuchinger, L.-S. Geng, and W. Weise, Phys. Rev. D 89, 014026 (2014).

[34] M.-L. Du, F.-K. Guo, U.-G. Meißner, and D.-L. Yao, Eur. Phys. J. C 77, 728 (2017).

[35] T. N. Truong, Phys. Rev. Lett. 61, 2526 (1988).

[36] J. R. Peláez, Phys. Rep. 658, 1 (2016).

[37] J. Gasser and U. G. Meissner, Nucl. Phys. B357, 90 (1991).

[38] P. C. Bruns and M. Mai, Phys. Lett. B 778, 43 (2018).

[39] M. Albaladejo, P. Fernandez-Soler, F.-K. Guo, and J. Nieves, Phys. Lett. B 767, 465 (2017).

[40] G. Moir, M. Peardon, S. M. Ryan, C. E. Thomas, and D. J. Wilson, J. High Energy Phys. 10 (2016) 011.

[41] M. Neubert, Phys. Rep. 245, 259 (1994).

[42] C. B. Lang, D. Mohler, S. Prelovsek, and R. M. Woloshyn, Phys. Lett. B 750, 17 (2015).

[43] G. S. Bali, S. Collins, A. Cox, and A. Schäfer, Phys. Rev. D 96, 074501 (2017).

[44] D. Wilson (private communication).

[45] C. Hanhart, J. R. Peláez, and G. Ríos, Phys. Lett. B 739, 375 (2014).

[46] C. Hanhart, J. R. Peláez, and G. Ríos, Phys. Rev. Lett. 100, 152001 (2008).

[47] M. Albaladejo and J. A. Oller, Phys. Rev. D 86, 034003 (2012).

[48] R. Aaij et al. (LHCb Collaboration), Phys. Rev. D 94, 072001 (2016).

[49] M. J. Savage and M. B. Wise, Phys. Rev. D 39, 3346 (1989); 40, 3127(E) (1989).

[50] J. A. Oller and E. Oset, Phys. Rev. D 60, 074023 (1999).

[51] R. Aaij et al. (LHCb Collaboration), Phys. Rev. D 90, 072003 (2014).

[52] S. Weinberg, Phys. Rev. 137, B672 (1965).

[53] D. Mohler, C. B. Lang, L. Leskovec, S. Prelovsek, and R. M. Woloshyn, Phys. Rev. Lett. 111, 222001 (2013).

[54] A. Martínez Torres, E. Oset, S. Prelovsek, and A. Ramos, J. High Energy Phys. 05 (2015) 153.

[55] M. Albaladejo, D. Jido, J. Nieves, and E. Oset, Eur. Phys. J. C 76, 300 (2016).

[56] F.-K. Guo and U.-G. Meißner, Phys. Rev. D 84, 014013 (2011).

[57] M. F. M. Lutz and M. Soyeur, Nucl. Phys. A813, 14 (2008). 